\title{
Effectiveness of a modified 6-Fr endoscopic nasobiliary drainage catheter for patients with preoperative perihilar cholangiocarcinoma
}

\section{(ㄷ)(i) $\odot$}

\author{
Authors \\ Hiroki Kawashima', Yoshiki Hirooka², Eizaburo Ohno', Takuya Ishikawa', Ryoji Miyahara', Osamu Watanabe', \\ Kazuhiko Hayashi ${ }^{1}$, Masatoshi Ishigami ${ }^{1}$, Senju Hashimoto ${ }^{3}$, Tomoki Ebata ${ }^{4}$, Masato Nagino ${ }^{4}$, Hidemi Goto ${ }^{1}$
}

Institutions

1 Department of Gastroenterology and Hepatology, Nagoya University Graduate School of Medicine, Nagoya, Japan

2 Department of Endoscopy, Nagoya University Hospital, Nagoya, Japan

3 Department of Liver, Biliary Tract and Pancreas Diseases, Fujita Health University Hospital, Toyoake, Japan

4 Division of Surgical Oncology, Department of Surgery, Nagoya University Graduate School of Medicine, Nagoya, Japan

submitted 9.1.2018

accepted after revision 12.4 .2018

Bibliography

DOI https://doi.org/10.1055/a-0614-2202 |

Endoscopy International Open 2018; 06: E1020-E1030

(c) Georg Thieme Verlag KG Stuttgart · New York

ISSN 2364-3722

Corresponding author

Yoshiki Hirooka, Department of Endoscopy, Nagoya

University Hospital, 65 Tsuruma-cho, Showa-ku, Nagoya

466-8550, Japan

Fax: $+81-52-735-8860$

hirooka@med.nagoya-u.ac.jp

\section{ABSTRACT}

Background and study aims To decrease complications associated with preoperative endoscopic nasobiliary drainage (ENBD) for perihilar cholangiocarcinoma patients, we developed a modified 6-Fr ENBD catheter with multiple side holes (m-ENBD). The aim of this retrospective study was to compare the $\mathrm{m}$-ENBD catheter with a conventional 7-Fr ENBD catheter (c-ENBD).

Patients and methods This study involved 371 patients with suspected perihilar cholangiocarcinoma who underwent ENBD using a c-ENBD catheter or an m-ENBD catheter. The effectiveness of each catheter and the incidence of complications were evaluated. Univariate and multivariate analyses followed by propensity score matching were performed.

Results In 145 patients with total bilirubin levels $\geq 2.0 \mathrm{mg} /$ $\mathrm{dL}$ prior to drainage, these levels decreased to $<2.0 \mathrm{mg} / \mathrm{dL}$ after ENBD in $81.1 \%$ of the c-ENBD patients and in $74.0 \%$ of the $\mathrm{m}$-ENBD patients $(P=0.325)$. Post-ENBD cholangitis occurred in $24.9 \%$ of the c-ENBD patients and in $12.4 \%$ of the m-ENBD patients $(P=0.006)$. After propensity score matching, the rate of post-ENBD cholangitis $(P=0.007)$ and the number of patients requiring subsequent or additional drainage $(P=0.030)$ were significantly lower in the m-ENBD group.

Conclusion The modified 6-Fr ENBD catheter was associated with a lower incidence of post-ENBD cholangitis than the conventional 7-Fr ENBD catheter, and the incidence of subsequent or additional drainage procedures was also decreased.

UMIN-CTR

UMIN000025631

TRIAL REGISTRATION: Retrospective study of clinical utility of Endoscopic Retrograde Cholangiopancreatography and relative for digestive diseases.

UMIN-CTR (UMIN000025631)

\section{Introduction}

In Japan, preoperative drainage is reported to be useful for patients who require extended hepatectomy because this procedure reduces mortality and morbidity rates in patients with perihilar cholangiocarcinoma [1-5]. Although it has been re- ported that external drainage by percutaneous transhepatic biliary drainage (PTBD) is more suitable for preventing infectious complications than internal drainage by endoscopic biliary stenting (EBS) [6], PTBD catheter tract recurrence has been detected in $5.2 \%$ of patients with cholangiocarcinoma who undergo surgical resection [7], and it was reported that 


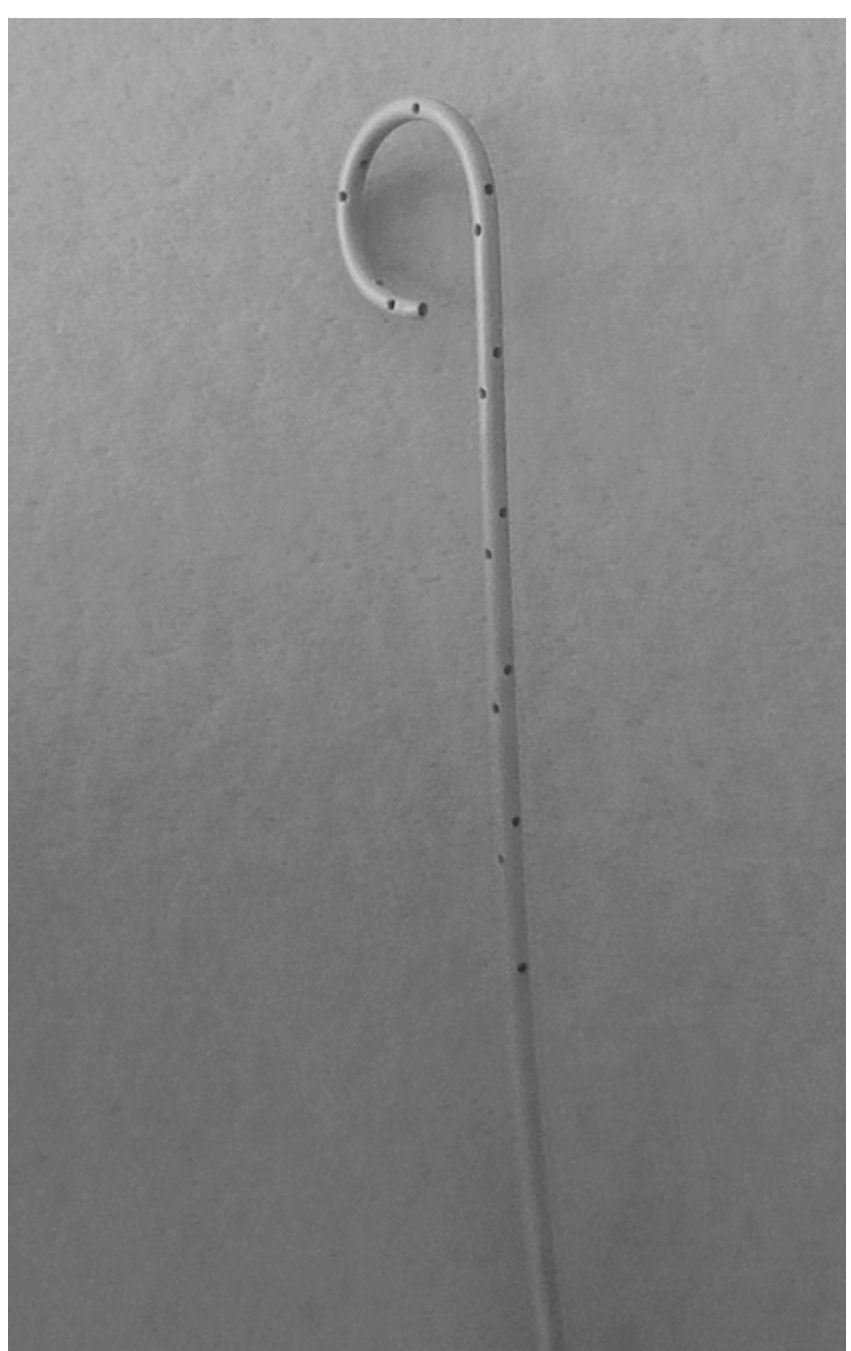

- Fig. 1 Newly developed catheter for endoscopic nasobiliary drainage. The catheter diameter is 6-Fr, and the multiple side holes are spiral-shaped with a dull bending pigtail-shaped tip.

PTBD was oncologically inferior to endoscopic nasobiliary drainage (ENBD) [8,9]. These results suggest that EBS and PTBD should be avoided in patients with biliary carcinoma. Thus, placing an ENBD in the future remnant lobe(s) is a feasible method for preoperative drainage in patients with perihilar cholangiocarcinoma [5, $10-12]$.

We previously reported the effectiveness of ENBD and the risk factors for complications associated with ENBD in preoperative perihilar cholangiocarcinoma patients [5]. In that study, rates of post-ENBD cholangitis and pancreatitis were $28.8 \%$ and $20.1 \%$, respectively. To reduce the incidence of these complications, we developed a modified pigtail-shaped 6-Fr ENBD catheter (m-ENBD; ENBD Catheter [GADELIUS MEDICAL K.K., Tokyo, Japan], \ Fig. 1) with multiple side holes and a dull bending pigtail-shaped tip, because the conventional 7- $\mathrm{Fr}$ ENBD catheter (c-ENBD; Nasal Biliary Drainage Catheter [COOK Medical, Winston-Salem, North Carolina, United States]), which is widely used in Japan, has a sharp bending pigtail-shaped tip that tended to become wedged in the thin biliary branch, lead- ing to poor drainage and post-ENBD cholangitis. Furthermore, the 6-Fr catheter, which is thinner than the 7-Fr catheter, might reduce incidence of post-ENBD pancreatitis [13].

We conducted this retrospective single-center study to examine the effectiveness of $m$-ENBD compared with c-ENBD among patients with preoperative suspected perihilar cholangiocarcinoma, many of whom met the Bismuth-Corlette criteria (B-C) for type III-IV tumours [14], using a propensity score matching analysis.

\section{Patients and methods}

\section{Patients}

Preoperative biliary drainage was performed in 517 consecutive patients with suspected operable perihilar cholangiocarcinoma based on multidetector-row computed tomography (MDCT) findings $[15,16]$ at Nagoya University Hospital between January 2007 and March 2016. Of these patients, this retrospective study included the patients who underwent successful ENBD (i.e., an ENBD catheter was inserted into the bile duct of the future remnant lobe) using a c-ENBD catheter or an m-ENBD catheter for comparison of these catheters. The following patients were excluded from this study: 113 patients (21.9\%) who underwent drainage using a 5-Fr ENBD catheter because two branches required drainage (76 patients) or the stricture site was too thin (37 patients), making the insertion of a $6-\mathrm{Fr}$ or 7 -Fr catheter difficult; 10 patients $(1.9 \%)$ who underwent drainage using another type of catheter; 1 patient $(0.2 \%)$ who was unable to undergo endoscopic retrograde cholangiography (ERC); and 22 patients (4.3\%) who could not have an ENBD catheter inserted into the future remnant lobe(s). These 23 patients who could not undergo ENBD catheter placement underwent PTBD. Overall, 371 patients were included in this retrospective study (c-ENBD group: 242 patients; m-ENBD group: 129 patients). Of these patients, 148 (39.9\%) were naïve patients (i.e., without previous drainage or endoscopic sphincterotomy [EST]), and the remaining 223 patients had previously undergone biliary drainage, EST, or both (150 EBS patients, 65 ENBD patients, and 125 EST patients) ( $\triangleright$ Fig. 2$)$. The tumors were classified as B-C types I $(n=39)$, II $(n=50)$, IIIa $(n=110)$, IIIb $(n=47)$, and IV $(n=125)$ based on the MDCT findings. A total of 145 (39.1\%) patients had a total serum bilirubin (TB) value $\geq 2 \mathrm{mg} / \mathrm{dL}$ prior to receiving ENBD at our institution ( $>$ Table 1).

\section{Methods}

After the optimal surgical plan was formulated based on the MDCT findings, selective ERC of the future remnant lobe(s) was performed. When we could not decide the appropriate type of surgical procedure, we inserted two 5-Fr ENBD catheters into two branches. For patients who had not undergone biliary drainage at a previous hospital, preoperative ENBD was indicated when the intrahepatic bile duct of the future remnant lobe was dilated, regardless of presence of jaundice. When a patient had previously undergone effective drainage, ERC was performed to confirm the feasibility of the planned surgical procedure using a biliary forceps biopsy for confirmation that 
Suspected perihilar cholangiocarcinoma $(n=517)$

Excluded patients $(n=146)$

- using 5-Fr ENBD catheter $(n=113)$

- using another type of catheter $(n=10)$

- ERC failure $(n=1)$

- insertion into not FRL branch $(n=22)$

Successful ENBD using c-ENBD or m-ENBD catheter $(n=371)$

\section{$\sqrt{ }$}

c-ENBD group $(n=242)$

- naive papilla ( $\mathrm{n}=91)$

- post EST or EBD $(n=151)$

Clinical outcome

- surgical operation $(n=164)$

L2 $(n=30)$,

$\mathrm{L} 2+\mathrm{PD}(\mathrm{n}=4)$

L3 $(n=35)$,

$\mathrm{L} 3+\mathrm{PD}(\mathrm{n}=4)$

R2 ( $n=47)$,

$\mathrm{R} 2+\mathrm{PD}(\mathrm{n}=17)$

R3 $(n=13)$,

$R 3+P D(n=3)$

others $(n=11)$

- inoperable $(n=72)$

- benignancy $(n=6)$

$\downarrow$

m-ENBD group $(n=129)$

- naive papilla ( $n=57)$

- post EST or EBD $(n=72)$

Clinical outcome
- surgical operation
$(n=94)$
L2 $(n=17)$,
L2 + PD $(n=6)$
L3 $(n=20)$,
L3 + PD $(n=2)$
R2 $(n=25)$,
R2 + PD $(n=12)$
R3 $(n=7)$,
R3 + PD $(n=0)$
others $(n=5)$
- inoperable $(n=32)$
- benignancy $(n=3)$

Fig. 2 Flow chart of patients. This retrospective study included patients who underwent successful ENBD (i. e., an ENBD catheter was inserted into the bile duct of the future remnant lobe) using a c-ENBD or an m-ENBD catheter for comparison of these catheters. Six patients with benign disease underwent surgery. ENBD, endoscopic nasobiliary drainage; ERC, endoscopic retrograde cholangiography; FRL, future remnant lobe(s); c-ENBD, conventionalENBD; $m$-ENBD, modified ENBD; EST, endoscopic sphincterotomy; $E B D$, endoscopic biliary drainage; L2, left hepatectomy; L3, left hepatic trisectionectomy; R2, right hepatectomy; R3, right hepatic trisectionectomy; PD, pancreatoduodenectomy

there was no surface extension that could not be diagnosed by imaging studies; the ENBD catheter was then reinserted into the bile duct of the future remnant lobe. A c-ENBD catheter was primarily used from January 2007 to March 2012, and the m-ENBD catheter was primarily used from April 2012 to March 2016. To ensure that all of the side holes of the catheter were upstream of the stricture site, the m-ENBD catheter was inserted upstream of the bile duct ( $\triangleright$ Fig. 3 ). However, no difference was observed between the c-ENBD and $\mathrm{m}$-ENBD groups with regard to methods of catheter insertion. Catheter insertion was performed without an EST after assessing for malignancy and the longitudinal and lateral tumor progression via intraductal ultrasonography (IDUS) [17] and a biliary forceps biopsy [18]. The ENBD catheters were inserted into the left bile duct for a right hepatectomy, into the right bile duct for a left hepatectomy, into the left lateral sectional bile duct for a right hepatic trisectionectomy, and into the right posterior sectional bile duct for a left hepatic trisectionectomy [19]. Preoperative bile replacement was performed during the external drainage, as previously reported [20]. In all patients with previous EBS (inserted into future remnant lobe in 65 patients; inserted into a lobe that was not the future remnant lobe in 66 patients; and inserted into both lobes in 19 patients), the stents were removed, and the ENBD catheters were inserted. In the patients whose first unilateral ENBD was not sufficient, a subsequent ENBD or PTBD was performed. In the patients with segmental cholangitis who did not improve with conservative treatment within 12 hours, the ENBD catheter was replaced or an additional ENBD or PTBD was inserted into the undrained bile duct. When patients were diagnosed as inoperable, the ENBD catheters were removed and internal drainage using a plastic stent or a self-expandable metal stent was performed.

The endoscopes used were a JF $260 \mathrm{~V}$ and a TJF $260 \mathrm{~V}$ (Olympus Medical, Tokyo, Japan), and the guide wires used were Jagwire Plus (Boston Scientific, Natick, Massachusetts, United States), VisiGlide (Olympus Medical, Tokyo, Japan) and RADIFOCUS (Terumo, Tokyo, Japan).

This study was approved by the Nagoya University Hospital institutional review board (No. 2016-0032) and was performed in accordance with the Declaration of Helsinki.

\section{Definitions}

In patients with a TB value $\geq 2 \mathrm{mg} / \mathrm{dL}$ prior to receiving ENBD at our institution, an effective first ENBD was defined as a TB value that decreased to $<2.0 \mathrm{mg} / \mathrm{dL}$ without additional drainage. The final diagnosis was obtained either from the surgical specimen or after more than 3 months of follow-up.

Catheter dislocation was defined as a decrease in the amount of bile, which was monitored every 6 hours, due to dislocation of the tip of the catheter downstream of the stricture site. Catheter impaction was defined as a decrease in the amount of bile due to impaction of the catheter tip into a thin branch ( $\triangleright$ Fig.3b). Cholangitis was defined according to the 2013 Tokyo Guidelines' diagnostic criteria (satisfied with the suspected diagnosis criteria) [21]. Post-ENBD pancreatitis was defined according to the 2012 revision of the Atlanta classification and definitions [22].

\section{Statistical analyses}

Statistical calculations were performed using SPSS 24.0 (SPSS, Chicago, Illinois, United States). The analyses were performed using the Mann-Whitney $U$ test for continuous variables and the chi-square test and Fisher's exact test for categorical variables. Continuous parameters were presented as medians (ranges). Factors with $P$ values $<0.1$ in the univariate analysis were included in a multiple logistic regression analysis.

After comparing the clinical data between the c-ENBD and m-ENBD groups, adjustments for significant differences in baseline characteristics between the 2 groups were performed 
Table 1 Clinical features according to the type of drainage catheter (including all 371 patients).

\begin{tabular}{|c|c|c|c|}
\hline & c-ENBD (7-Fr) & m-ENBD (6-Fr) & \\
\hline Variables & $(n=242)$ & $(n=129)$ & $P$ value \\
\hline \multirow[t]{2}{*}{ Age, y (range) } & & & 0.756 \\
\hline & $69(34-83)$ & $68(35-90)$ & \\
\hline Gender (\%) & & & 0.100 \\
\hline - Male & $148(61.2)$ & $90(69.8)$ & \\
\hline - Female & $94(38.8)$ & $39(30.2)$ & \\
\hline \multicolumn{2}{|l|}{ Bismuth-Corlette criteria, n (\%) } & & 0.040 \\
\hline - I, II & $50(20.7)$ & $39(28.1)$ & \\
\hline . III, IV & $192(79.3)$ & $90(69.8)$ & \\
\hline Pre-ENBD cholangitis (\%) & & & 0.512 \\
\hline . With & $34(14.0)$ & $15(11.6)$ & \\
\hline - Without & $208(86.0)$ & $114(88.4)$ & \\
\hline \multicolumn{2}{|l|}{ Pre-ENBD total serum bilirubin (\%) } & & 0.926 \\
\hline - $\geq 2 \mathrm{mg} / \mathrm{dL}$ & $95(39.3)$ & $50(38.8)$ & \\
\hline . $<2 \mathrm{mg} / \mathrm{dL}$ & $147(60.7)$ & $79(61.2)$ & \\
\hline Previous EBS (\%) & & & 0.356 \\
\hline - With & $102(42.1)$ & $48(37.2)$ & \\
\hline - Without & $140(57.9)$ & $81(62.8)$ & \\
\hline Previous ENBD (\%) & & & 0.863 \\
\hline . With & $43(17.8)$ & $22(17.1)$ & \\
\hline - Without & $199(82.2)$ & 107 (82.9) & \\
\hline Previous EST (\%) & & & 0.570 \\
\hline . With & $84(34.7)$ & $41(31.8)$ & \\
\hline . Without & $158(65.3)$ & $88(68.2)$ & \\
\hline Pancreatography (\%) & & & 0.029 \\
\hline - Present & $91(37.6)$ & $34(26.4)$ & \\
\hline - Absent & $151(62.4)$ & $95(73.6)$ & \\
\hline \multicolumn{2}{|l|}{ Duration of ENBD, d (range) } & & 0.039 \\
\hline & $29(2-96)$ & $33(3-144)$ & \\
\hline Catheter dislocation (\%) & & & 0.073 \\
\hline - Occurred & $6(2.5)$ & $8(6.2)$ & \\
\hline - Not occurred & $236(97.5)$ & $121(93.8)$ & \\
\hline \multicolumn{2}{|l|}{ Catheter impaction (\%) } & & 0.071 \\
\hline - Occurred & $19(7.9)$ & $4(3.1)$ & \\
\hline - Not occurred & $223(92.1)$ & 125 (96.9) & \\
\hline \multicolumn{2}{|l|}{ Subsequent and/or additional drainage } & & 0.123 \\
\hline - Required & $58(24.0)$ & $22(17.1)$ & \\
\hline - Not required & $184(76.0)$ & 107 (82.9) & \\
\hline
\end{tabular}


- Table 1 (Continuation)

\begin{tabular}{|l|c|c|}
\hline & c-ENBD (7-Fr) & m-ENBD (6-Fr) \\
\hline Variables & $\mathbf{( n = 2 4 2 )}$ & $\mathbf{( n = 1 2 9 )}$ \\
\hline \begin{tabular}{l} 
Post-ENBD cholangitis (\%) \\
\hline - With
\end{tabular} & $59(24.4)$ & $16(12.4)$ \\
\hline - Without & $183(75.6)$ & $113(87.6)$ \\
\hline Post-ENBD pancreatitis (\%) & & \\
\hline - With & $39(16.1)$ & $21(16.3)$ \\
\hline - Without & $203(83.9)$ & $108(83.7)$ \\
\hline $\begin{array}{l}\text { ENBD, endoscopic nasobiliary drainage; c-ENBD, conventional ENBD catheter; m-ENBD, modified ENBD catheter; EBS, endoscopic biliary stenting; EST, endoscopic } \\
\text { sphincterotomy }\end{array}$ \\
\hline
\end{tabular}
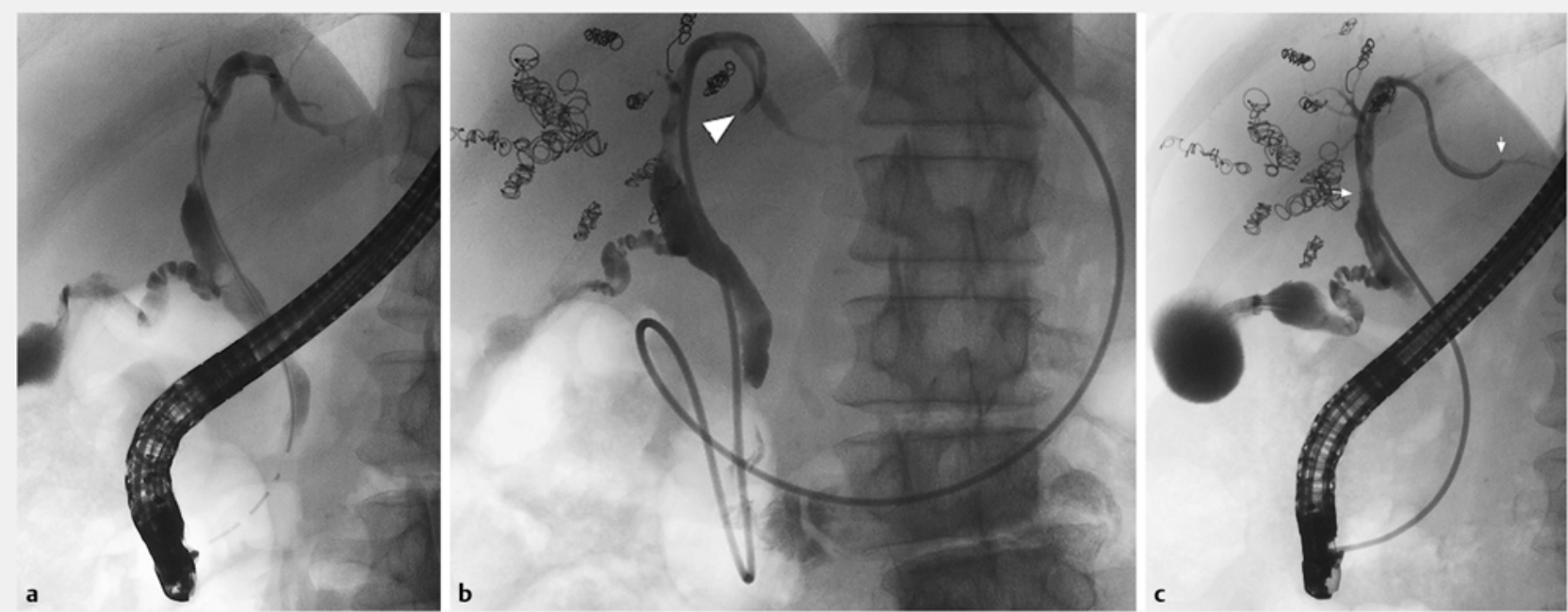

- Fig. 3 Endoscopic nasobiliary drainage (ENBD) for perihilar cholangiocarcinoma. a The endoscopic retrograde cholangiography findings showed a Bismuth-Corlette type IIla perihilar cholangiocarcinoma. Because the optimal surgical plan involved a right trisectionectomy, a conventional 7-Fr ENBD catheter was inserted into the left lateral sectional bile duct. $\mathbf{b}$ After 20 days, the amount of bile flow from the ENBD catheter was decreased. Cholangiography via the ENBD catheter showed that the catheter tip was impacted in the thin side branch (arrow head).c A modified 6-Fr ENBD catheter was inserted upstream of the bile duct so that all of the side holes were upstream of the stricture site (arrows).

with a propensity score matching analysis [23-25]. Propensity scores were estimated using a multivariate logistic regression analysis, and the following six variables that might have affected incidence of post-ENBD complications were used in the model: gender, B-C type (I-II/ III-IV), with/without pre-ENBD cholangitis, previous EST, previous transpapillary drainage (EBS or ENBD) and presence/absence of pancreatography. Subsequently, a one-to-one match between the two groups was performed using the nearest-neighbor matching method within 0.05 standard deviations. A $P$ value $<0.05$ was considered significant.

\section{Results}

\section{Effectiveness of ENBD}

Median duration of ENBD placement was 29 days (2-96 days) in the c-ENBD group and 33 days (3-144 days) in the m-ENBD group; this difference was significant $(P=0.039)$. Catheter dislocation occurred in six patients in the c-ENBD group and nine patients in the m-ENBD group; this difference was not significant $(P=0.071)$. Catheter impaction occurred in 19 patients in the c-ENBD group and four patients in the m-ENBD group; this difference was not significant $(P=0.071$; $>$ Table 1$)$. A final diagnosis of malignant disease was reached in 356 patients (302 patients with perihilar cholangiocarcinoma, 52 patients with gallbladder carcinoma and two patients with hepatocellular carcinoma), and a benign diagnosis was reached in 15 patients. 
- Table 2 Univariate and multivariate analyses of predictive factors of ENBD effectiveness.

\begin{tabular}{|c|c|c|c|c|c|}
\hline & ENBD effectiveness & & Univariate & Multivariate & \\
\hline Variables & Presence $(n=114)$ & Absence $(n=31)$ & $P$ & OR (95\% CI) & $P$ \\
\hline \multirow[t]{2}{*}{ Age, y (range) } & & & 0.324 & & \\
\hline & $68(34-89)$ & $70(50-90)$ & & & \\
\hline Gender & & & 0.535 & & \\
\hline - Male & 78 & 23 & & & \\
\hline - Female & 36 & 8 & & & \\
\hline Bismuth-Corlette criteria, $\mathrm{n}$ & & & 0.245 & & \\
\hline - I, II & 21 & 3 & & & \\
\hline - III, IV & 93 & 28 & & & \\
\hline Pre-ENBD cholangitis & & & 0.301 & & \\
\hline - With & 20 & 8 & & & \\
\hline - Without & 94 & 23 & & & \\
\hline \multicolumn{3}{|c|}{ Pre-ENBD total serum bilirubin value (range) (mg/dL) } & $<0.001$ & $1.11(1.05-1.18)$ & $<0.001$ \\
\hline & $3.9(1.9-32.1)$ & $13.75(3.1-33.1)$ & & & \\
\hline Previous EBS & & & 0.993 & & \\
\hline - With & 33 & 9 & & & \\
\hline - Without & 81 & 22 & & & \\
\hline Previous EST & & & 0.09 & & 0.107 \\
\hline - With & 36 & 5 & & & \\
\hline - Without & 78 & 26 & & & \\
\hline Post ENBD cholangitis & & & 0.233 & & \\
\hline - With & 25 & 10 & & & \\
\hline - Without & 89 & 21 & & & \\
\hline ENBD catheter & & & 0.325 & & \\
\hline - c-ENBD (7Fr) & 77 & 18 & & & \\
\hline - m-ENBD (6Fr) & 37 & 13 & & & \\
\hline \multirow[t]{2}{*}{ Duration of ENBD, d (range) } & & & 0.033 & & 0.120 \\
\hline & $37(3-144)$ & $24(3-96)$ & & & \\
\hline PVE & & & $<0.001$ & & 0.001 \\
\hline . With & 77 & 10 & & 1 & \\
\hline - Without & 37 & 21 & & $4.60(1.82-11.6)$ & \\
\hline
\end{tabular}

ENBD, endoscopic nasobiliary drainage; EBS, endoscopic biliary stenting; EST, endoscopic sphincterotomy; c-ENBD, conventional ENBD catheter; m-ENBD, modified ENBD catheter: PVE, portal vein embolization

Finally, a hemihepatectomy or a more extended resection was performed in 258 patients. The remaining 98 patients with malignancies who did not undergo hepatectomy underwent chemoradiotherapy, chemotherapy, or supportive care.

Of the 145 patients with TB values $\geq 2.0 \mathrm{mg} / \mathrm{dL}$ prior to drainage, the first unilateral ENBD using a c-ENBD catheter was effective in $77 / 95$ (81.1\%) patients, and the m-ENBD catheter was effective in $37 / 50(74.0 \%)$ patients; this difference was not significant $(P=0.325)$. The significant negative predictive factors for ENBD effectiveness according to the multivariate analysis included an elevated TB level prior to ENBD $(P<0.001$, odds ratio $[95 \% \mathrm{Cls}]=1.11[1.05-1.18])$ and lack of portal vein embolization (PVE) $(P=0.001$, odds ratio $[95 \% \mathrm{Cls}]=4.60$ $[1.82-11.6])$ ( Table 2 ). Duration of ENBD was significantly longer in the effective ENBD group in univariate analysis. It was thought that this results was affected by inoperable pa- 
- Table 3 Univariate and multivariate analyses of post-ENBD cholangitis.

\begin{tabular}{|c|c|c|c|c|c|}
\hline \multirow[b]{2}{*}{ Variables } & \multicolumn{2}{|l|}{ Cholangitis } & \multirow{2}{*}{$\begin{array}{l}\text { Univariate } \\
P\end{array}$} & \multicolumn{2}{|l|}{ Multivariate } \\
\hline & With $(n=75)$ & Without $(n=296)$ & & OR (95\% Cl) & $P$ \\
\hline \multicolumn{2}{|l|}{ Age, y (range) } & & 0.611 & & \\
\hline & $68.0(34-83)$ & $68.5(35-90)$ & & & \\
\hline Gender & & & 0.029 & & 0.057 \\
\hline - Male & 40 & 198 & & & \\
\hline - Female & 35 & 98 & & & \\
\hline \multicolumn{2}{|l|}{ Bismuth-Corlette criteria } & & 0.016 & & 0.082 \\
\hline - I, II & 10 & 79 & & & \\
\hline - III, IV & 65 & 217 & & & \\
\hline \multicolumn{2}{|l|}{ Pre-ENBD cholangitis } & & 0.002 & & 0.003 \\
\hline . With & 18 & 31 & & $2.77(1.40-5.46)$ & \\
\hline - Without & 57 & 265 & & 1 & \\
\hline \multicolumn{2}{|l|}{ Pre-ENBD total serum bilirubin } & & 0.132 & & \\
\hline - $\geq 2 \mathrm{mg} / \mathrm{dL}$ & 35 & 110 & & & \\
\hline . $<2 \mathrm{mg} / \mathrm{dL}$ & 40 & 186 & & & \\
\hline \multicolumn{2}{|l|}{ Previous EBS } & & 0.005 & & 0.175 \\
\hline - With & 41 & 109 & & & \\
\hline - Without & 34 & 187 & & & \\
\hline \multicolumn{2}{|l|}{ Previous EST } & & $<0.001$ & & 0.002 \\
\hline " With & 38 & 87 & & $2.31(1.35-3.95)$ & \\
\hline - Without & 37 & 209 & & 1 & \\
\hline \multicolumn{2}{|l|}{ Pancreatography } & & 0.636 & & \\
\hline - Present & 27 & 98 & & & \\
\hline - Absent & 48 & 198 & & & \\
\hline ENBD & & & 0.006 & & 0.002 \\
\hline - c-ENBD (7-Fr) & 59 & 183 & & $2.76(1.45-5.26)$ & \\
\hline " m-ENBD (6-Fr) & 16 & 113 & & 1 & \\
\hline \multicolumn{2}{|l|}{ Duration of ENBD, $d$ (range) } & & 0.007 & $1.02(1.01-1.03)$ & 0.002 \\
\hline & $36(5-96)$ & $29(2-144)$ & & & \\
\hline
\end{tabular}

ENBD, endoscopic nasobiliary drainage; EBS, endoscopic biliary stenting; EST, endoscopic sphincterotomy; c-ENBD, conventional ENBD catheter; m-ENBD, modified ENBD catheter

tients who were switched to another drainage method before ENBD was effective.

\section{Complication rate and risk factors}

Post-ENBD cholangitis occurred in 59/242 (24.9\%) patients in the c-ENBD group and in $16 / 129(12.4 \%)$ patients in the $\mathrm{m}$ ENBD group; this difference was significant $(P=0.006$; $>$ Table 1). According to multivariate analysis, significant risk factors for post-ENBD cholangitis were previous ENBD cholangitis $(P=0.003$, odds ratio $[95 \% \mathrm{Cls}]=2.77[1.40-5.46] ;>$ Table 3$)$, previous EST $(P=0.002$, odds ratio $[95 \% \mathrm{Cls}]=2.31[1.35-$ 3.95]; $>$ Table 3), use of a c-ENBD catheter $(P=0.002$, odds ratio $[95 \% \mathrm{Cls}]=2.76[1.45-5.26]$; $>$ Table 3$)$ and a longer duration of ENBD $(P=0.002$, odds ratio $[95 \% \mathrm{Cls}]=1.02[1.01-$ 1.03]; - Table 3).

There was no significant difference $(P=0.968)$ in the occurrence rate for post-ENBD pancreatitis between the c-ENBD group (39/242 [16.1\%]; mild: 33 patients; moderate: four patients; and severe: two patients) and the m-ENBD group (21/ 129 [16.3\%]; mild: 20 patients and moderate: one patient). All 
- Table4 Univariate and multivariate analyses of post-ENBD pancreatitis.

\begin{tabular}{|c|c|c|c|c|c|}
\hline \multirow[b]{2}{*}{ Variables } & \multicolumn{2}{|l|}{ Pancreatitis } & \multirow{2}{*}{$\begin{array}{l}\text { Univariate } \\
P\end{array}$} & \multicolumn{2}{|l|}{ Multivariate } \\
\hline & With $(n=60)$ & Without $(n=311)$ & & OR $(95 \% \mathrm{Cl})$ & $P$ \\
\hline \multicolumn{2}{|l|}{ Age, y (range) } & & 0.083 & & 0.101 \\
\hline & $70.5(50-84)$ & $68(34-90)$ & & & \\
\hline Gender & & & 0.885 & & \\
\hline - Male & 38 & 200 & & & \\
\hline - Female & 22 & 111 & & & \\
\hline \multicolumn{2}{|c|}{ Bismuth-Corlette criteria } & & 0.429 & & \\
\hline - I, II & 12 & 77 & & & \\
\hline - III, IV & 48 & 234 & & & \\
\hline \multicolumn{6}{|c|}{ Pre-ENBD cholangitis } \\
\hline - With & 4 & 45 & & & \\
\hline - Without & 56 & 266 & & & \\
\hline \multicolumn{2}{|c|}{ Pre-ENBD total serum bilirubin } & & 0.198 & & \\
\hline - $\geq 2 \mathrm{mg} / \mathrm{dL}$ & 19 & 126 & & & \\
\hline - $<2 \mathrm{mg} / \mathrm{dL}$ & 41 & 185 & & & \\
\hline \multicolumn{2}{|c|}{ Previous EBS or ENBD } & & $<0.001$ & & $<0.001$ \\
\hline - With & 7 & 196 & & 1 & \\
\hline - Without & 53 & 115 & & $9.00(3.88-20.9)$ & \\
\hline \multicolumn{2}{|l|}{ Previous EST } & & $<0.001$ & & 0.095 \\
\hline - With & 5 & 120 & & & \\
\hline - Without & 55 & 191 & & & \\
\hline \multicolumn{2}{|l|}{ Pancreatography } & & $<0.001$ & & $<0.001$ \\
\hline - Present & 42 & 83 & & $3.90(2.04-7.43)$ & \\
\hline - Absent & 18 & 228 & & 1 & \\
\hline ENBD & & & 0.968 & & \\
\hline - c-ENBD (7-Fr) & 39 & 203 & & & \\
\hline - m-ENBD (6-Fr) & 21 & 108 & & & \\
\hline
\end{tabular}

of the patients with post-ENBD pancreatitis were cured using conservative treatment (i. e., fasting, transfusions, and antibiotics). According to multivariate analysis, significant risk factors for post-ENBD pancreatitis included undergoing pancreatography $(P<0.001$, odds ratio [95\% Cls] = 9 [2.04- 7.43]; > Table 4) and absence of previous EBS or ENBD ( $P<0.001$, odds ratio [95\% Cls] $=3.90$ [3.88-20.9]; $>$ Table 4).

Replacement of the ENBD catheter and additional ENBD or PTBD procedures due to poor drainage, cholangitis, or both were required in 58/242 patients (24.0\%) in the c-ENBD group and in 22/129 patients (17.1\%) in the m-ENBD group; however, this difference was not significant $(P=0.123$; $>$ Table 1$)$. Significant risk factors for required subsequent and/or additional drainage in the multivariate analysis were longer duration of ENBD $(P=0.001$, odds ratio $[95 \% \mathrm{Cls}]=1.021[1.009-1.033])$ and pre-ENBD cholangitis $(P=0.002$, odds ratio $[95 \% \mathrm{Cls}]=$ 2.810 [1.451-5.439]).

Overall, only a single endoscopic session achieved effective drainage in $179 / 242$ patients $(74.0 \%)$ in the c-ENBD group and $98 / 129$ patients $(76.0 \%)$ in the m-ENBD group without moderate or severe pancreatitis, cholangitis requiring subsequent/ additional drainage, or both. This difference was not significant $(P=0.673)$. 
- Table 5 Clinical features according to type of drainage catheter after propensity score matching (including 118 patients in each group).

\begin{tabular}{|c|c|c|c|}
\hline & c-ENBD (7-Fr) & m-ENBD (6-Fr) & \\
\hline Variables & $(n=118)$ & $(n=118)$ & $P$ \\
\hline \multirow[t]{2}{*}{ Age, y (range) } & & & 0.773 \\
\hline & $69(45-83)$ & $69.5(35-90)$ & \\
\hline Gender (\%) & & & 1.000 \\
\hline - Male & $81(68.6)$ & $81(68.6)$ & \\
\hline - Female & $37(31.4)$ & $37(31.4)$ & \\
\hline \multicolumn{2}{|l|}{ Bismuth-Corlette criteria, $\mathrm{n}(\%)$} & & 1.000 \\
\hline - I, II & $31(26.3)$ & $31(26.3)$ & \\
\hline - III, IV & $87(73.7)$ & $87(73.7)$ & \\
\hline Pre-ENBD cholangitis (\%) & & & 1.000 \\
\hline - With & $10(8.5)$ & $10(8.5)$ & \\
\hline - Without & $108(91.5)$ & $108(91.5)$ & \\
\hline \multicolumn{2}{|l|}{ Pre-ENBD total serum bilirubin (\%) } & & 0.894 \\
\hline - $\geq 2 \mathrm{mg} / \mathrm{dL}$ & $47(39.8)$ & $41(34.7)$ & \\
\hline - $<2 \mathrm{mg} / \mathrm{dL}$ & $71(60.2)$ & $77(65.3)$ & \\
\hline Previous EBS (\%) & & & 0.684 \\
\hline - With & $44(37.3)$ & $41(34.7)$ & \\
\hline - Without & $74(62.7)$ & $77(65.3)$ & \\
\hline Previous ENBD (\%) & & & 0.717 \\
\hline - With & $17(14.4)$ & $19(16.1)$ & \\
\hline - Without & $101(85.6)$ & $99(83.9)$ & \\
\hline Previous EST (\%) & & & 1.000 \\
\hline - With & $34(28.8)$ & $34(28.8)$ & \\
\hline - Without & $84(71.2)$ & $84(71.2)$ & \\
\hline Pancreatography (\%) & & & 1.000 \\
\hline - Present & $33(28.0)$ & $33(28.0)$ & \\
\hline - Absent & $85(72.0)$ & $85(72.0)$ & \\
\hline \multicolumn{2}{|l|}{ Duration of ENBD, d (range) } & & 0.183 \\
\hline & $29(2-95)$ & $33(3-144)$ & \\
\hline Catheter dislocation (\%) & & & 0.308 \\
\hline - Occurred & $3(2.5)$ & $6(5.1)$ & \\
\hline - Not occurred & $115(97.5)$ & $112(94.9)$ & \\
\hline Catheter impaction (\%) & & & 0.098 \\
\hline - Occurred & $10(8.5)$ & $4(3.4)$ & \\
\hline - Not occurred & $108(91.5)$ & $114(96.6)$ & \\
\hline \multicolumn{2}{|l|}{ Subsequent and/or additional drainage (\%) } & & 0.030 \\
\hline - Required & $34(28.8)$ & $20(16.9)$ & \\
\hline - Not required & $84(71.2)$ & $98(83.1)$ & \\
\hline
\end{tabular}


Table 5 (Continuation)

\begin{tabular}{|l|l|l|}
\hline & C-ENBD (7-Fr) & m-ENBD (6-Fr) \\
\hline Variables & $\mathbf{( n = 1 1 8 )}$ & $\mathbf{( n = 1 1 8 )}$ \\
\hline \begin{tabular}{l} 
Post-ENBD cholangitis (\%) \\
\hline - With
\end{tabular} & $30(25.4)$ & $14(11.9)$ \\
\hline - Without & $88(74.6)$ & $104(88.1)$ \\
\hline Post-ENBD pancreatitis (\%) & & \\
\hline - With & $19(16.1)$ & $19(16.1)$ \\
\hline - Without & $99(83.9)$ & $99(83.9)$ \\
\hline $\begin{array}{l}\text { ENBD, endoscopic nasobiliary drainage; c-ENBD, conventional ENBD catheter; m-ENBD, modified ENBD catheter; EBS, endoscopic biliary stenting; EST, endoscopic } \\
\text { sphincterotomy }\end{array}$ \\
\hline
\end{tabular}

\section{Propensity score matching analysis}

Baseline characteristics of the new cohort after matching (118 patients in each group) were similar between the two groups $(\triangleright$ Table 5). In the new cohort, the post-ENBD cholangitis rate $(P=0.007)$ and subsequent and/or additional drainage rate $(P$ $=0.030$ ) were significantly lower in the m-ENBD group. The rate of catheter impaction tended to be lower in the m-ENBD group, but this difference was not significant $(P=0.098)$.

\section{Discussion}

Due to recent advances in perioperative management, surgical outcomes in patients with perihilar cholangiocarcinoma have improved [26,27]. Biliary drainage and PVE [28] play pivotal roles as preoperative intervention procedures. Preoperative biliary drainage is performed to promote early bile duct decompression in future remnant lobe(s), improve liver function and prevent cholangitis. Because conservative therapy cannot improve obstructive jaundice, drainage procedures should be performed as soon as possible.

ENBD is advantageous because drainage and diagnosis can both be achieved in a single session, and ENBD catheters were successfully inserted in the targeted bile ducts of $>90 \%$ of patients, including many with B-C type III-IV tumors. In cases in which the catheter was successfully inserted in the future remnant lobe(s), a drainage effect was achieved in approximately $80 \%$ of patients using unilateral ENBD [5]. However, ENBD has several disadvantages, such as post-procedural cholangitis and/or pancreatitis and patient discomfort. Though ENBD sometimes causes electrolyte imbalances due to body fluid loss, no patients had electrolyte imbalances in this study, because preoperative bile replacement was performed during the external drainage in our hospital.

Catheter diameter most likely affects the effectiveness of drainage as well as complication rates and patients' comfort. It has been reported that the rate of decreases in bilirubin was significantly higher when a 7-Fr ENBD catheter was used than when a 5-Fr ENBD catheter was used, but patient discomfort was significantly greater when a 7-Fr ENBD catheter was used
[29]. On the other hand, in a comparison study of 4-Fr versus 6 -Fr ENBD catheters, there was no significant difference in ENBD effectiveness [13]. ENBD catheters with thinner diameters might induce less effective drainage. In the current study, no significant difference in effectiveness of drainage between the c-ENBD (7-Fr in a diameter) and m-ENBD (6-Fr in a diameter) groups was observed. The multiple side holes in the $\mathrm{m}$ ENBD catheter most likely contributed to effective drainage. The dull bending pigtail-shaped tip of the m-ENBD catheter also decreased the occurrence of catheter impaction that causes poor drainage and post-ENBD cholangitis. The rate of postENBD cholangitis was significantly lower in the m-ENBD group, and the incidence of catheter impaction tended to be lower in the $\mathrm{m}$-ENBD group according to the propensity score matching analysis. On the other hand, the dull bending tip tended to increase incidence of catheter dislocation but not to a significant degree. These results suggest that the requirement for subsequent/additional drainage could be significantly lower when using the m-ENBD catheter.

It has been reported that prevalence of post-ENBD pancreatitis was significantly lower when a 4-Fr ENBD catheter was used than when a 6-Fr catheter was used [13]. Although the 6-Fr catheter was expected to reduce incidence of post-ENBD pancreatitis in this study, no significant differences were found with regard to incidence of post-ENBD pancreatitis between the c-ENBD and m-ENBD groups, and the only significant risk factor for post-ENBD pancreatitis that could be avoided was presence of pancreatography. Although prevalence of postENBD pancreatitis was high (approximately $16 \%$ ) in this study, there was fortunately only one patient (in the c-ENBD group with severe post-ENBD pancreatitis) who could not undergo radical surgery due to pancreatitis. In addition, no patients in the $m$-ENBD group had severe post-ENBD pancreatitis. The thinner diameter of the ENBD catheter might reduce severity of postENBD pancreatitis. However, post-ENBD pancreatitis is the most common complication to be avoided, and additional devices should be evaluated to reduce incidence of post-ENBD pancreatitis. Although EST is often added to prevent post-ENBD pancreatitis, this procedure was an independent risk factor for post-ENBD cholangitis and was not a significant risk factor for 
post-ENBD pancreatitis in this study. These results corroborate the findings from our previous study [5]. Therefore, EST should be avoided to prevent post-ENBD cholangitis when ENBD is administered for perihilar cholangiocarcinoma because preoperative cholangitis is a potential cause of post-hepatectomy liver failure [30]. The current study has three limitations: retrospective design; the difference among the periods when the ENBD catheter was primarily used; and inclusion of many patients with a TB value $<2.0 \mathrm{mg} / \mathrm{dL}$. These limitations may have affected the results.

\section{Conclusion}

In conclusion, use of a modified 6-Fr ENBD catheter with multiple side holes and a dull bending pigtail-shaped tip was associated with a lower incidence of post-ENBD cholangitis than use of a 7-Fr ENBD catheter, and use of this catheter could decrease the subsequent incidence of and need for additional drainage procedures.

\section{Competing interests}

The Department of Gastroenterology and Hepatology, Nagoya University Graduate School of Medicine, received scholarship donations from GADELIUS MEDICAL K.K.

\section{References}

[1] Nagino M, Nimura Y, Kamiya et al. Preoperative management of hilar cholangiocarcinoma. J Hepatobiliary Pancreat Surg 1995; 2: 215-223

[2] Nimura Y, Kamiya J, Kondo S et al. Aggressive preoperative management and extended surgery for hila cholangiocarcinoma: Nagoya experience. J Hepatobiliary Pancreat Surg 2000; 7: 155-162

[3] Nagino M, Takada T, Miyazaki M et al. Preoperative biliary drainage for biliary tract and ampullary carcinomas. J Hepatobiliary Pancreat Surg 2008; 15: 25-30

[4] Nagino M, Kamiya J, Nishio $\mathrm{H}$ et al. Two hundred forty consecutive portal vein embolizations before extended hepatectomy for biliary cancer surgical outcome and long-term follow-up. Ann Surg 2006; 243: $364-372$

[5] Kawashima H, Itoh A, Ohno E et al. Preoperative endoscopic nasobiliary drainage in 164 consecutive patients with suspected perihilar cholangiocarcinoma: a retrospective study of efficacy and risk factors related to complications. Ann Surg 2013; 257: 121 - 127

[6] Kloek JJ, van der Gaag NA, Aziz Y et al. Endoscopic and percutaneous preoperative biliary drainage in patients with suspected hilar cholangiocarcinoma. J Gastrointest Surg 2010; 14: 119-125

[7] Takahashi Y, Nagino M, Nishio H et al. Percutaneous transhepatic biliary drainage catheter tract recurrence in cholangiocarcinoma. $\mathrm{Br}$ J Surg 2010; 97: $1860-1866$

[8] Komaya K, Ebata T, Fukami Y et al. Percutaneous biliary drainage is oncologically inferior to endoscopic drainage: a propensity score matching analysis in resectable distal cholangiocarcinoma. J Gastroenterol 2016; 51: 608-619

[9] Komaya K, Ebata T, Yokoyama Y et al. Verification of the oncologic inferiority of percutaneous biliary drainage to endoscopic drainage: A propensity score matching analysis of resectable perihilar cholangiocarcinoma. Surgery 2017; 161: 394-404
[10] Maguchi H, Takahashi K, Katanuma A et al. Preoperative biliary drainage for hilar cholangiocarcinoma. J Hepatobiliary Pancreat Surg 2007; 14: $441-446$

[11] Arakura N, Takayama M, Ozaki Y et al. Efficacy of preoperative endoscopic nasobiliary drainage for hilar cholangiocarcinoma. J Hepatobiliary Pancreat Surg 2009; 16: $473-477$

[12] Kawakami H, Kuwatani M, Onodera M et al. Endoscopic nasobiliary drainage is the most suitable preoperative biliary drainage method in the management of patients with hilar cholangiocarcinoma. J Gastroenterol 2011; 46: $242-248$

[13] Ishigaki T, Sasaki T, Serikawa M et al. Comparative study of $4 \mathrm{Fr}$ versus $6 \mathrm{Fr}$ nasobiliary drainage catheters: a randomized, controlled trial. J Gastroenterol Hepatol 2014; 29: 653-659

[14] Bismuth H, Corlette MB. Intrahepatic cholangioenteric anastomosis in carcinoma of the hilus of the liver. Surg Gynecol Obstet 1975; 140: $170-176$

[15] Sugiura T, Nishio H, Nagino M et al. Value of multidetector-row computed tomography in diagnosis of portal vein invasion by perihilar cholangiocarcinoma. World J Surg 2008; 32: 1478-1484

[16] Senda $\mathrm{Y}$, Nishio $\mathrm{H}$, Oda $\mathrm{K}$ et al. Value of multidetector row $\mathrm{CT}$ in the assessment of longitudinal extension of cholangiocarcinoma - correlation between MDCT and microscopic findings. World J Surg 2009; 33: $1459-1467$

[17] Kawashima H, Hirooka Y, Itoh A et al. Progress of endoscopic ultrasonography and intraductal ultrasonography in the diagnosis of malignant biliary diseases. J Hepatobiliary Pancreat Surg 2006; 13: 69 - 74

[18] Kawashima $\mathrm{H}$, Itoh A, Ohno E et al. Diagnostic and prognostic value of immunohistochemical expression of S100P and IMP3 in transpapillary biliary forceps biopsy samples of extrahepatic bile duct carcinoma. J Hepatobiliary Pancreat Sci 2013; 20: $441-447$

[19] Natsume S, Ebata T, Yokoyama Y et al. Clinical significance of left trisectionectomy for perihilar cholangiocarcinoma: An appraisal and complication with left hepatectomy. Ann Surg 2012; 255: 754-762

[20] Kamiya S, Nagino M, Kanazawa $\mathrm{H}$ et al. The value of bile replacement during external biliary drainage: an analysis of intestinal permeability, integrity, and microflora. Ann Surg 2004; 239: 510-517

[21] Kiriyama S, Takada T, Strasberg SM et al. New diagnostic criteria and severity assessment of acute cholangitis in revised Tokyo guidelines. J Hepatobiliary Pancreat Sci 2012; 19: 548- 556

[22] Banks PA, Bollen TL, Dervenis C et al. Classification of acute pancreatitis-2012: revision of the Atlanta classification and definitions by international consensus. Gut 2013; 62: 102-111

[23] Rosenbaum PR. Model-based direct adjustment. J AM Stat Assoc 1987; 82: $387-394$

[24] Rubin DB. Estimating causal effects from large data sets using propensity scores. Ann Intern Med 1997; 127: 757-763

[25] Austin PC. An introduction to propensity score methods for reducing the effects of confounding in observational studies. Multivar Behav Res 2011; 46: 399-424

[26] Igami T, Nishio H, Ebata T et al. Surgical treatment of hilar cholangiocarcinoma in the "new era": the Nagoya University experience. J Hepatobiliary Pancreat Sci 2010; 17: 449-454

[27] Ebata T, Yokoyama Y, Igami T et al. Hepatopancreatoduodenectomy for cholangiocarcinoma: a single-center review of 85 consecutive patients. Ann Surg 2012; 256: 297 - 305

[28] Ebata T, Yokoyama Y, Igami T et al. Portal vein embolization before extended hepatectomy for biliary cancer: current technique and review of 494 consecutive embolizations. Dig Surg 2012; 29: 23-29

[29] Fujisawa T, Kagawa K, Watanabe $S$ et al. Endoscopic nasobiliary drainage for obstructive jaundice using either a $5 \mathrm{Fr}$ or $7 \mathrm{Fr}$ catheter: a prospective, randomized trial. BMC Gastroenterol 2014; 14: 161

[30] Nagino M, Nimura Y, Hayakawa N et al. Logistic regression and discriminant analyses of hepatic failure after liver resection for carcinoma of the biliary tract. World J Surg 1993; 17: $250-255$ 